\title{
EXTENSIONS OF MULTILINEAR OPERATORS AND BANACH SPACE PROPERTIES
}

\author{
JOAQUÍN M. GUTIÉRREZ AND IGNACIO VILLANUEVA
}

\begin{abstract}
A new characterization of the Dunford-Pettis property in terms of the extensions of multilinear operators to the biduals is obtained. For the first time, multilinear characterizations of the reciprocal Dunford-Pettis property and Pełczyński's property (V) are also found. Polynomial and holomorphic versions of these properties are given as well.
\end{abstract}

\section{INTRODUCTION}

Given a $k$-linear operator $T: E_{1} \times \cdots \times E_{k} \rightarrow X$ between Banach spaces, Aron and Berner [3] introduced a procedure to extend it to an operator $\bar{T}: E_{1}^{* *} \times \cdots \times E_{k}^{* *} \rightarrow$ $X^{* *}$ between the biduals. In the linear case $T: E \rightarrow X$, this extension is unique and coincides with the bitranspose $T^{* *}: E^{* *} \rightarrow X^{* *}$ of $T$.

Many Banach space properties may be defined in terms of linear operators. Various authors have tried to express these properties in terms of multilinear operators and polynomials. This has been achieved, e.g., for the Dunford-Pettis property [30] and the noncontainment of $\ell_{1}[5,17]$. However, the properties given by the fact that the linear operators of certain class have to be weakly compact do not have an equivalent multilinear version: this is the case of the reciprocal Dunford-Pettis property and Pełczyński's property (V) [21]. This is why it has been said that the weak compactness of a multilinear operator or a polynomial should be redefined so that the linear and multilinear properties may be equivalent.

In this paper we show that this can be done by replacing weak compactness of a multilinear operator $T$ by the fact that the Aron-Berner extension of $T$ be $X$-valued. In the linear case, by Gantmacher's theorem [25, §2.18], the bitranspose of $T$ is $X$ valued if and only if $T$ is weakly compact. For $k>1$, the Aron-Berner extension of a weakly compact $k$-linear operator is $X$-valued; there are, however, many non weakly compact $k$-linear operators with $X$-valued Aron-Berner extension.

1991 Mathematics Subject Classification. Primary 46G25; Secondary 46B20, 47H60.

Key words and phrases. Multilinear operator, polynomial, holomorphic mapping, Aron-Berner extension, (reciprocal) Dunford-Pettis property, property (V).

The first named author was supported in part by Dirección General de Investigación, BFM 2000-0609 (Spain).

The second named author was partially supported by Dirección General de Investigación, BFM 2001-1284 (Spain).

file abext.tex. 
In this way, we obtain a new multilinear characterization of the Dunford-Pettis property, and give, for the first time, multilinear, polynomial and holomorphic characterizations of the reciprocal Dunford-Pettis property and Pełczyński's property $(\mathrm{V})$. The starting point of this research were the second named author's results in [34] for multilinear operators on $C(K)$ spaces. Some of our proofs refine in different ways an idea of Ryan's [30]. The fact of having an $X$-valued Aron-Berner extension was already used, instead of weak compactness, in [22] in order to give a polynomial characterization of the Grothendieck property.

Throughout, $E, E_{1}, \ldots, E_{k}$ and $X$ denote Banach spaces, $E^{*}$ is the dual of $E$, $B_{E}$ is the closed unit ball of $E$, and $\omega^{*}$ represents the weak-star topology on a dual Banach space. For a subset $A \subset E, \bar{\Gamma}(A)$ stands for the absolutely convex closed hull of $A$. All the operators and polynomials are supposed to be continuous. The notation $\mathcal{L}^{k}\left(E_{1}, \ldots, E_{k} ; X\right)$ will be used for the space of all $k$-linear operators from $E_{1} \times \cdots \times E_{k}$ into $X ; \mathcal{L}_{s}^{k}(E, X)$ denotes the space of symmetric $k$-linear operators from $E^{k}$ into $X$, and $\mathcal{P}\left({ }^{k} E, X\right)$ stands for the $k$-homogeneous polynomials from $E$ into $X$. Whenever the range space $X$ is omitted, it is understood to be the scalar field. The space of linear operators from $E$ into $X$ is denoted by $\mathcal{L}(E, X)$, and its subspace of weakly compact operators is represented by $\mathcal{L}_{\text {wco }}(E, X)$. All these spaces are endowed with the usual operator norm.

To each $P \in \mathcal{P}\left({ }^{k} E, X\right)$ we can associate a unique $T \in \mathcal{L}_{s}^{k}(E, X)$ so that $P(x)=$ $T(x, \ldots, x)$ for each $x \in E$. For the general theory of multilinear operators, polynomials and holomorphic mappings, we refer to $[15,27]$.

The paper is organized as follows. In Section 2 we introduce the definition and main properties of the Aron-Berner extensions; Section 3 is devoted to the properties related to the completely continuous operators, namely, the Dunford-Pettis and the reciprocal Dunford-Pettis property; Section 4 deals with the unconditionally converging operators and hereby with property (V); Section 5 extends the results to the holomorphic setting; Section 6 raises the question of characterizing the multilinear operators whose extensions are $X$-valued. At the beginning of each section, the necessary definitions are recalled.

\section{Aron-Berner extensions}

We start by introducing the Aron-Berner extension of an arbitrary multilinear operator, since the original extension considered in [3] was meant only for symmetric operators.

We first consider scalar-valued operators. Let $1 \leq m \leq k$. For fixed $1 \leq i_{1}<$ $\cdots<i_{m} \leq k$ and $z \in E_{i_{m}}^{* *}$, using the notation of [36], we define the linear operator

$$
\bar{z}: \mathcal{L}^{m}\left(E_{i_{1}}, \ldots, E_{i_{m}}\right) \longrightarrow \mathcal{L}^{m-1}\left(E_{i_{1}}, \ldots, E_{i_{m-1}}\right)
$$

by

$$
\bar{z}(S)\left(x_{i_{1}}, \ldots, x_{i_{m-1}}\right)=\left\langle z, S_{x_{i_{1}}, \ldots, x_{i_{m-1}}}\right\rangle
$$


where $x_{i_{j}} \in E_{i_{j}}(1 \leq j \leq m-1), S \in \mathcal{L}^{m}\left(E_{i_{1}}, \ldots, E_{i_{m}}\right)$, and $S_{x_{i_{1}}, \ldots, x_{i_{m-1}}} \in E_{i_{m}}^{*}$ is given by

$$
\left\langle S_{x_{i_{1}}, \ldots, x_{i_{m-1}}}, x\right\rangle=S\left(x_{i_{1}}, \ldots, x_{i_{m-1}}, x\right) \quad\left(x \in E_{i_{m}}\right) .
$$

Then to each $T \in \mathcal{L}^{k}\left(E_{1}, \ldots, E_{k}\right)$ we associate $\bar{T} \in \mathcal{L}^{k}\left(E_{1}^{* *}, \ldots, E_{k}^{* *}\right)$ given by

$$
\bar{T}\left(z_{1}, \ldots, z_{k}\right)=\overline{z_{1}} \circ \cdots \circ \overline{z_{k}}(T) \text { for all } z_{i} \in E_{i}^{* *} \quad(1 \leq i \leq k),
$$

where we have identified $\mathcal{L}^{0}(E)$ with the scalar field. Clearly, $\bar{T}$ is an extension of $T$, and $\|\bar{T}\|=\|T\|$. We could also have defined

$$
\bar{T}\left(z_{1}, \ldots, z_{k}\right)=\overline{z_{i_{1}}} \circ \cdots \circ \overline{z_{i_{k}}}(T)
$$

where $\left\{i_{1}, \ldots, i_{k}\right\}$ is a permutation of the indices $\{1, \ldots, k\}$. Therefore it is possible to have $k$ ! extensions of $T$ which may be different. For simplicity, we shall use the same notation $\bar{T}$ for any of these Aron-Berner extensions of $T$. If $E_{1}=\cdots=E_{k}$ and $T$ is symmetric, then $\bar{T}\left(z_{1}, \ldots, z_{k}\right)$ will exactly mean the right hand side of $(2)$ while we shall denote by $\bar{T}\left(z_{i_{1}}, \ldots, z_{i_{k}}\right)$ the right hand side of (3) since $\bar{T}$ need not be symmetric. We shall refer to (2), i.e., to the extension carried out from the last variable to the first, as the Davie-Gamelin extension of $T$ [11].

We now consider vector-valued multilinear mappings. Let $T \in \mathcal{L}^{k}\left(E_{1}, \ldots, E_{k} ; X\right)$ be given. An operator $\bar{T} \in \mathcal{L}^{k}\left(E_{1}^{* *}, \ldots, E_{k}^{* *} ; X^{* *}\right)$ will be said to be an Aron-Berner extension of $T$ if it satisfies

$$
\left\langle\bar{T}\left(z_{1}, \ldots, z_{k}\right), \psi\right\rangle=\overline{\psi \circ T}\left(z_{1}, \ldots, z_{k}\right) \quad\left(\psi \in X^{*}\right)
$$

where $\overline{\psi \circ T}$ is a fixed Aron-Berner extension of $\psi \circ T$.

If the chosen extension is exactly $\overline{\psi \circ T}\left(z_{1}, \ldots, z_{k}\right)=\overline{z_{i_{1}}} \circ \cdots \circ \overline{z_{i_{k}}}(\psi \circ T)$, then we have the equality $\bar{T}\left(z_{1}, \ldots, z_{k}\right)=\overline{z_{i_{1}}} \circ \cdots \circ \overline{z_{i_{k}}}(T)$ where, for each $m \in\{1, \ldots, k\}$ and $z_{i_{m}} \in E_{i_{m}}^{* *}$, the operator

$$
\overline{z_{i_{m}}}: \mathcal{L}^{m}\left(E_{i_{1}}, \ldots, E_{i_{m}} ; X^{* *}\right) \longrightarrow \mathcal{L}^{m-1}\left(E_{i_{1}}, \ldots, E_{i_{m-1}} ; X^{* *}\right)
$$

is given by

$$
\left\langle\overline{z_{i_{m}}}(S)\left(x_{i_{1}}, \ldots, x_{i_{m-1}}\right), \psi\right\rangle=\overline{z_{i_{m}}}(\psi \circ S)\left(x_{i_{1}}, \ldots, x_{i_{m-1}}\right)
$$

for each $S \in \mathcal{L}^{m}\left(E_{i_{1}}, \ldots, E_{i_{m}} ; X^{* *}\right)$. We use the same notation for the operator in (1) and in (4) since no confusion seems possible.

As in the scalar-valued case, we can get $k$ ! extensions of $T$ which may be different. Again the Davie-Gamelin extension of $T$ will be the one obtained when we proceed from the last variable to the first.

The following lemma will be frequently used in the sequel.

Lemma 2.1. Let $T \in \mathcal{L}^{k}\left(E_{1}, \ldots, E_{k} ; X\right)$. Then:

(a) Given $i, j \in\{1, \ldots, k\}(i \neq j), z_{j} \in E_{j}^{* *}$ and $x_{i} \in E_{i}$, we have

$$
\overline{x_{i}} \circ \overline{z_{j}}(T)=\overline{z_{j}} \circ \overline{x_{i}}(T) \text {. }
$$

(b) Given $1 \leq m \leq k, 1 \leq i_{1}<\cdots<i_{m} \leq k, x_{i_{j}} \in E_{i_{j}}(1 \leq j \leq m)$, we have

$$
\overline{x_{i_{1}}} \circ \cdots \circ \overline{x_{i_{m}}}(T)=T_{x_{i_{1}}, \ldots, x_{i_{m}}} \text {. }
$$


(c) Fixed $i \in\{1, \ldots, k\}, x_{1} \in E_{1}, \ldots, x_{i-1} \in E_{i-1}, z_{i+1} \in E_{i+1}^{* *}, \ldots, z_{k} \in E_{k}^{* *}$, if $\bar{T}$ denotes the Davie-Gamelin extension of $T$, then the linear operator

$$
z_{i} \in E_{i}^{* *} \longmapsto \bar{T}\left(x_{1}, \ldots, x_{i-1}, z_{i}, \ldots, z_{k}\right) \in X^{* *}
$$

is $\omega^{*}-\omega^{*}$ continuous. The same is true, with obvious modifications in the choice and order of the variables, for any other Aron-Berner extension of T. As a consequence, every Aron-Berner extension $\bar{T}$ of $T$ is $\omega^{*}-\omega^{*}$ continuous in the last extended variable.

(d) For $z_{i} \in E_{i}^{* *}(2 \leq i \leq k)$ fixed, suppose that $\overline{z_{2}} \circ \cdots \circ \overline{z_{k}}(T)$ is $X$-valued, and define $S \in \mathcal{L}\left(E_{1}, X\right)$ by

$$
S\left(x_{1}\right)=\overline{x_{1}} \circ \overline{z_{2}} \circ \cdots \circ \overline{z_{k}}(T)=\bar{T}\left(x_{1}, z_{2}, \ldots, z_{k}\right) .
$$

Then

$$
S^{* *}\left(z_{1}\right)=\overline{z_{1}} \circ \cdots \circ \overline{z_{k}}(T)=\bar{T}\left(z_{1}, \ldots, z_{k}\right) \quad \text { for all } z_{1} \in E_{1}^{* *} .
$$

Proof. Parts (a) and (b) follow the steps of [35, Proposition 2], (c) is essentially contained in [18, Corollary 5.2], and (d) is immediate using the end of (c).

If $P \in \mathcal{P}\left({ }^{k} E, X\right)$ and $T$ is the associated symmetric operator, the Aron-Berner extension of $P$ is the polynomial $\bar{P} \in \mathcal{P}\left({ }^{k} E^{* *}, X^{* *}\right)$ defined by

$$
\bar{P}(z)=\bar{T}(z, \ldots, z) \quad\left(z \in E^{* *}\right) .
$$

Note that the Aron-Berner extension of a polynomial is unique, since all the AronBerner extensions of $T$ coincide on the diagonal.

There is a particular case when all the Aron-Berner extensions of a multilinear operator coincide. The following result is mentioned in $[4, \S 8]$ in the scalar-valued case:

Proposition 2.2. Let $T \in \mathcal{L}^{k}\left(E_{1}, \ldots, E_{k} ; X\right)$. If $T$ has a separately $\omega^{*}-\omega^{*}$ continuous extension $\widetilde{T} \in \mathcal{L}^{k}\left(E_{1}^{* *}, \ldots, E_{k}^{* *} ; X^{* *}\right)$, then every Aron-Berner extension of $T$ coincides with $\widetilde{T}$.

\section{Completely continuous operators}

In this Section, we consider the Banach space properties that relate weakly compact and completely continuous linear operators, namely, the Dunford-Pettis and the reciprocal Dunford-Pettis property, obtaining multilinear characterizations of both.

We say that $T \in \mathcal{L}^{k}\left(E_{1}, \ldots, E_{k} ; X\right)$ is completely continuous, and we write $T \in$ $\mathcal{L}_{\text {cc }}^{k}\left(E_{1}, \ldots, E_{k} ; X\right)$, if, given weak Cauchy sequences $\left(x_{i}^{n}\right)_{n \in \mathbb{N}} \subset E_{i}(1 \leq i \leq k)$, the sequence $\left(T\left(x_{1}^{n}, \ldots, x_{k}^{n}\right)\right)_{n}$ is norm convergent in $X$. This definition may be adapted to polynomials in an obvious way. The space of completely continuous polynomials is denoted by $\mathcal{P}_{\mathrm{cc}}\left({ }^{k} E, X\right)$. By the polarization formula [27, Theorem 1.10], a polynomial is completely continuous if and only if so is its associated symmetric multilinear operator.

Recall that $E$ has the Dunford-Pettis property (DPP, for short) if, for every $X$, $\mathcal{L}_{\text {wco }}(E, X) \subseteq \mathcal{L}_{\text {cc }}(E, X)$. Examples of spaces with the DPP are $C(K)$ and $L_{1}(\mu)$ 
spaces. $E$ has the reciprocal Dunford-Pettis property (RDPP, for short) if, for every $X, \mathcal{L}_{\text {cc }}(E, X) \subseteq \mathcal{L}_{\text {wco }}(E, X)$. The spaces containing no copy of $\ell_{1}$, and $C(K)$ spaces have the RDPP. Both properties were introduced in [24].

A subset $A \subset E$ is a Dunford-Pettis set (DP set, for short) if, for every weakly null sequence $\left(\phi_{n}\right) \subset E^{*}$, we have

$$
\lim _{n} \sup _{x \in A}\left|\phi_{n}(x)\right|=0 .
$$

We say that a sequence $\left(x_{n}\right) \subset E$ is a $D P$ sequence if the set $\left\{x_{n}: n \in \mathbb{N}\right\}$ is a DP set. We say that $\left(x_{n}\right) \subset E$ is a weak Cauchy DP sequence if it is both a weak Cauchy sequence and a DP sequence. It is easy to show that $E$ has the DPP if and only if every weak Cauchy sequence in $E$ is a DP sequence $[1, \S \mathrm{I}]$.

We first state a well-known, useful result, whose proof is contained in [5, Theorem 2.3 and Lemma 2.4].

Lemma 3.1. Let $T \in \mathcal{L}^{k}\left(E_{1}, \ldots, E_{k} ; X\right)$. Then $T$ is completely continuous if and only if, for all weak Cauchy sequences $\left(x_{j}^{n}\right)_{n \in \mathbb{N}} \subset E_{j}(1 \leq j \leq k)$, at least one of which converges weakly to zero, we have

$$
\lim _{n \rightarrow \infty}\left\|T\left(x_{1}^{n}, \ldots, x_{k}^{n}\right)\right\|=0 .
$$

The following technical lemma will be crucial to prove our first main result.

Lemma 3.2. Let $T \in \mathcal{L}^{k}\left(E_{1}, \ldots, E_{k} ; X\right)$. Then $T$ is completely continuous if and only if, for all weak Cauchy sequences $\left(x_{j}^{n}\right)_{n \in \mathbb{N}} \subset E_{j}(1 \leq j \leq k)$, at least one of which, say $\left(x_{i}^{n}\right)_{n}$, converges weakly to zero, we have

$$
\lim _{m \rightarrow \infty} \sup _{n \in \mathbb{N}}\left\|T\left(x_{1}^{n}, \ldots, x_{i-1}^{n}, x_{i}^{m}, x_{i+1}^{n}, \ldots, x_{k}^{n}\right)\right\|=0 .
$$

Proof. Let $T$ be completely continuous. If the conclusion does not follow, we can find $\epsilon>0$, weak Cauchy sequences $\left(x_{j}^{n}\right)_{n} \subset E_{j}(1 \leq j \leq k)$, and $i \in\{1, \ldots, k\}$ so that $\left(x_{i}^{n}\right)_{n}$ is weakly convergent to zero, and two increasing sequences of indices $(m(p))_{p \in \mathbb{N}},(n(p))_{p \in \mathbb{N}}$, in such a way that

$$
\left\|T\left(x_{1}^{n(p)}, \ldots, x_{i-1}^{n(p)}, x_{i}^{m(p)}, x_{i+1}^{n(p)}, \ldots, x_{k}^{n(p)}\right)\right\|>\epsilon \quad(p \in \mathbb{N}),
$$

which contradicts Lemma 3.1. The converse is clear.

If $X$ is a Banach space, $c(X)$ is defined as the closed subspace of $\ell_{\infty}(X)$ formed by the convergent sequences of elements of $X$. The following lemma is proved analogously to [30, Lemma 1.2].

Lemma 3.3. Let $S \in \mathcal{L}(E, c(X))$, with $S(x)=\left(S_{n}(x)\right)_{n}$. Then $S$ is weakly compact if and only if

(a) for every $n \in \mathbb{N}, S_{n} \in \mathcal{L}(E, X)$ is weakly compact, and

(b) for every $z \in E^{* *}$, the sequence $\left(S_{n}^{* *}(z)\right)_{n}$ converges.

Theorem 3.4. The following statements are equivalent:

(a) The spaces $E_{1}, \ldots, E_{k}$ have the RDPP; 
(b) for all $X$ and $T \in \mathcal{L}_{\mathrm{cc}}^{k}\left(E_{1}, \ldots, E_{k} ; X\right)$, every Aron-Berner extension of $T$ is $X$-valued;

(c) for all $X$, every $T \in \mathcal{L}_{\mathrm{cc}}^{k}\left(E_{1}, \ldots, E_{k} ; X\right)$ has an $X$-valued Aron-Berner extension.

Proof. (a) $\Rightarrow(\mathrm{b})$ : We proceed by induction on $k$. If $k=1$, the result is clear. Suppose it is true for all $(k-1)$-linear operators and take $T$ as in the statement. Fix an Aron-Berner extension $\bar{T}$ of $T$. Suppose, without loss of generality, that $\bar{T}$ is the Davie-Gamelin extension. For each $z_{k} \in E_{k}^{* *}$, consider $\overline{z_{k}}(T) \in$ $\mathcal{L}^{k-1}\left(E_{1}, \ldots, E_{k-1} ; X\right)$. To see that $\overline{z_{k}}(T)$ is indeed $X$-valued, observe that, if we fix $\left(x_{1}, \ldots, x_{k-1}\right) \in E_{1} \times \cdots \times E_{k-1}$, then $T_{x_{1}, \ldots, x_{k-1}} \in \mathcal{L}_{\mathrm{cc}}\left(E_{k}, X\right) \subseteq \mathcal{L}_{\text {wco }}\left(E_{k}, X\right)$. Therefore $\left(T_{x_{1}, \ldots, x_{k-1}}\right)^{* *} \in \mathcal{L}\left(E_{k}^{* *}, X\right)$ and, according to Lemma 2.1,

$$
\overline{z_{k}}(T)\left(x_{1}, \ldots, x_{k-1}\right)=\overline{z_{k}} \circ \overline{x_{1}} \circ \cdots \circ \overline{x_{k-1}}(T)=\left(T_{x_{1}, \ldots, x_{k-1}}\right)^{* *}\left(z_{k}\right) \in X .
$$

In order to show that $\overline{z_{k}}(T)$ is completely continuous, take weak Cauchy sequences $\left(x_{i}^{n}\right)_{n} \subset E_{i}(1 \leq i \leq k-1)$. We can define the operator $S \in \mathcal{L}\left(E_{k} ; c(X)\right)$ by

$$
S(x)=\left(S_{n}(x)\right)_{n} \quad \text { where } \quad S_{n}(x)=T\left(x_{1}^{n}, \ldots, x_{k-1}^{n}, x\right) \quad\left(x \in E_{k}\right) .
$$

By Lemma 3.2, $S$ is completely continuous, hence weakly compact. By Lemma 3.3, for every $z_{k} \in E_{k}^{* *}$, the sequence

$$
\left(\overline{z_{k}}(T)\left(x_{1}^{n}, \ldots, x_{k-1}^{n}\right)\right)_{n}=\left(S_{n}^{* *}\left(z_{k}\right)\right)_{n}
$$

is convergent in $X$. Therefore $\overline{z_{k}}(T)$ is completely continuous. By the induction hypothesis, all of its Aron-Berner extensions are $X$-valued, and we conclude that $\bar{T}$ is $X$-valued.

(b) $\Rightarrow$ (c) is clear.

$(c) \Rightarrow\left(\right.$ a): Suppose one of the spaces, say $E_{1}$ for simplicity, does not have the RDPP. Then there exist $X$ and a non weakly compact operator $U \in \mathcal{L}_{\mathrm{cc}}\left(E_{1}, X\right)$. For $2 \leq i \leq k$ choose a nonzero form $\phi_{i} \in E_{i}^{*}$. We can define $T \in \mathcal{L}^{k}\left(E_{1}, \ldots, E_{k} ; X\right)$ by

$$
T\left(x_{1}, \ldots, x_{k}\right)=\prod_{i=2}^{k} \phi_{i}\left(x_{i}\right) U\left(x_{1}\right) .
$$

Easily, $T$ is completely continuous. The only Aron-Berner extension of $T$ is given by $\bar{T}\left(z_{1}, \ldots, z_{k}\right)=\prod_{i=2}^{k}\left\langle z_{i}, \phi_{i}\right\rangle U^{* *}\left(z_{1}\right)$ and is clearly not $X$-valued.

Corollary 3.5. The following assertions are equivalent:

(a) The space $E$ has the RDPP;

(b) for all $X$ and $k \in \mathbb{N}$, the Aron-Berner extension of every $P \in \mathcal{P}_{\mathrm{cc}}\left({ }^{k} E, X\right)$ is $X$-valued;

(c) there is $k \in \mathbb{N}$ such that, for all $X$, the Aron-Berner extension of every $P \in \mathcal{P}_{\text {cc }}\left({ }^{k} E, X\right)$ is $X$-valued.

Proof. (a) $\Rightarrow(\mathrm{b})$ : Suppose $E$ has the RDPP, and let $P \in \mathcal{P}_{\mathrm{cc}}\left({ }^{k} E, X\right)$. The associated $T \in \mathcal{L}_{s}^{k}(E, X)$ is also completely continuous. Therefore, it is enough to apply Theorem 3.4.

(b) $\Rightarrow(\mathrm{c})$ is obvious. 
(c) $\Rightarrow$ (a): If $E$ does not have the RDPP, we can find $X$ and an operator $S \in$ $\mathcal{L}_{\text {cc }}(E, X)$ which is not weakly compact. Take $z \in E^{* *}$ such that $S^{* *}(z) \in X^{* *} \backslash X$, and $\phi \in E^{*}$ with $\langle z, \phi\rangle=1$. Define $P \in \mathcal{P}\left({ }^{k} E, X\right)$ by $P(x)=(\phi(x))^{k-1} S(x)$. Then $P$ is completely continuous, and $\bar{P}(z)=S^{* *}(z) \in X^{* *} \backslash X$.

As a kind of reciprocal to the results above, we will now show that every multilinear operator $T \in \mathcal{L}^{k}\left(E_{1}, \ldots, E_{k} ; X\right)$ with an $X$-valued Aron-Berner extension is continuous for some "weak" topology. This will easily give a multilinear version of the Dunford-Pettis property and, as shown in Section 4, that such an operator $T$ is unconditionally converging.

We say that an operator $T \in \mathcal{L}^{k}\left(E_{1}, \ldots, E_{k} ; X\right)$ is weakly continuous on DP sets (see [19]) if, given weak Cauchy DP sequences $\left(x_{i}^{n}\right)_{n} \subset E_{i}(1 \leq i \leq k)$, the sequence $\left(T\left(x_{1}^{n}, \ldots, x_{k}^{n}\right)\right)_{n}$ is norm convergent in $X$. This definition may be adapted to polynomials in an obvious way.

Proposition 3.6. Suppose that $T \in \mathcal{L}^{k}\left(E_{1}, \ldots, E_{k} ; X\right)$ has an $X$-valued AronBerner extension. Then $T$ is weakly continuous on DP sets.

Proof. For $k=1, T$ is a weakly compact linear operator, and it is easy to show that every weakly compact linear operator is weakly continuous on DP sets [19, Proposition 3.1]. Assume the result is true for all $(k-1)$-linear operators, and let $T$ be as in the statement. To simplify the notation, we suppose that the DavieGamelin extension $\bar{T}$ of $T$ is $X$-valued, slight modifications being needed otherwise. Take $z \in E_{k}^{* *}$ and consider the $(k-1)$-linear operator $\bar{z}(T) \in \mathcal{L}^{k-1}\left(E_{1}, \ldots, E_{k-1} ; X\right)$. Clearly, the Davie-Gamelin extension of $\bar{z}(T)$ to $E_{1}^{* *} \times \cdots \times E_{k-1}^{* *}$ is $X$-valued. By the induction hypothesis, $\bar{z}(T)$ is weakly continuous on DP sets. by

Let $\left(x_{i}^{n}\right)_{n} \subset E_{i}(1 \leq i \leq k)$ be weak Cauchy DP sequences. Define $S_{n} \in \mathcal{L}\left(E_{k}, X\right)$

$$
S_{n}(x)=T\left(x_{1}^{n}, \ldots, x_{k-1}^{n}, x\right)=\bar{x}(T)\left(x_{1}^{n}, \ldots, x_{k-1}^{n}\right) \quad\left(x \in E_{k}\right) .
$$

For each $x \in E_{k}$, the weak continuity of $\bar{x}(T)$ on DP sets assures the existence of $\lim _{n} S_{n}(x)$. Now, we define the linear operator

$$
\begin{aligned}
S: E_{k} & \longrightarrow c(X) \\
x & \longmapsto\left(S_{n}(x)\right)_{n} .
\end{aligned}
$$

Applying Lemma 2.1, we have

$$
S_{n}^{* *}(z)=\left(\overline{x_{1}^{n}} \circ \cdots \circ \overline{x_{k-1}^{n}}(T)\right)^{* *}(z)=\bar{z}(T)\left(x_{1}^{n}, \cdots, x_{k-1}^{n}\right) \in X \quad\left(z \in E_{k}^{* *}\right) .
$$

Since $\bar{z}(T)$ is weakly continuous on DP sets, the sequence $\left(S_{n}^{* *}(z)\right)_{n}$ converges. By Lemma 3.3, $S$ is weakly compact and hence weakly continuous on DP sets. Thus, $\left(S\left(x_{k}^{n}\right)\right)_{n}$ converges to some $w=\left(w_{m}\right)_{m} \in c(X)$ and we have

$$
\lim _{n}\left\|S_{n}\left(x_{k}^{n}\right)-w_{n}\right\| \leq \lim _{n} \sup _{m}\left\|S_{m}\left(x_{k}^{n}\right)-w_{m}\right\|=\lim _{n}\left\|S\left(x_{k}^{n}\right)-w\right\|=0 .
$$

Since the sequence $\left(w_{n}\right)$ is convergent in $X$, so is the sequence

$$
\left(T\left(x_{1}^{n}, \ldots, x_{k}^{n}\right)\right)_{n}=\left(S_{n}\left(x_{k}^{n}\right)\right)_{n} .
$$


We say that an operator $T \in \mathcal{L}^{k}\left(E_{1}, \ldots, E_{k} ; X\right)$ is weakly compact if the set $K=T\left(B_{E_{1}} \times \cdots \times B_{E_{k}}\right)$ is relatively weakly compact in $X$. If $T$ is weakly compact, then all its Aron-Berner extensions are $X$-valued. Indeed, for $x_{i} \in E_{i}(1 \leq i \leq k-1)$, given $z_{k} \in B_{E_{k}^{* *}}$, choose a net $\left(x_{k}^{\alpha}\right)_{\alpha} \subset B_{E_{k}}$ weak-star converging to $z_{k}$. Then

$$
\begin{aligned}
\bar{T}\left(x_{1}, \ldots, x_{k-1}, z_{k}\right) & =\text { weak-star- } \lim _{\alpha} T\left(x_{1}, \ldots, x_{k-1}, x_{k}^{\alpha}\right) \\
& =\text { weak- } \lim _{\alpha} T\left(x_{1}, \ldots, x_{k-1}, x_{k}^{\alpha}\right) \\
& \in \bar{K}^{w},
\end{aligned}
$$

where $\bar{K}^{w}$ is the weak closure of $K$. Analogously, we get $\bar{T}\left(x_{1}, \ldots, x_{k-2}, z_{k-1}, z_{k}\right) \in$ $\bar{K}^{w}$. Reiterating the process, we obtain

$$
\bar{T}\left(z_{1}, \ldots, z_{k}\right) \in \bar{K}^{w} \quad \text { for } \quad z_{j} \in B_{E_{j}^{* *}} \quad(1 \leq j \leq k) .
$$

This fact was proved in [9, Proposition 1.4] for weakly compact polynomials. From it, we get:

Theorem 3.7. The following assertions are equivalent:

(a) The spaces $E_{1}, \ldots, E_{k}$ have the DPP;

(b) for every $X$, if $T \in \mathcal{L}^{k}\left(E_{1}, \ldots, E_{k} ; X\right)$ has an $X$-valued Aron-Berner extension, then $T$ is completely continuous;

(c) for every $X$, every weakly compact $T \in \mathcal{L}^{k}\left(E_{1}, \ldots, E_{k} ; X\right)$ is completely continuous;

(d) same statement as (c) with $X=c_{0}$.

Proof. (a) $\Rightarrow$ (b): Since, for each $1 \leq i \leq k, E_{i}$ has the DPP, every weak Cauchy sequence in $E_{i}$ is a DP sequence. Then it is enough to apply Proposition 3.6.

(b) $\Rightarrow$ (c) is clear by the comment preceding this Theorem.

(c) $\Rightarrow$ (d) is obvious.

$(d) \Rightarrow\left(\right.$ a): Suppose one of the spaces, say $E_{1}$ for simplicity, does not have the DPP. Then we can find an operator $S \in \mathcal{L}_{\text {wco }}\left(E_{1}, c_{0}\right)$ which is not completely continuous. Choose $\phi_{i} \in E_{i}^{*}(2 \leq i \leq k)$ with $\left\|\phi_{i}\right\|=1$. Define $T\left(x_{1}, \ldots, x_{k}\right)=\prod_{i=2}^{k} \phi_{i}\left(x_{i}\right) S\left(x_{1}\right)$ for $x_{i} \in E_{i}(1 \leq i \leq k)$. Clearly, $T$ is weakly compact. Choose a weakly null sequence $\left(x_{1}^{n}\right)_{n} \subset E_{1}$ so that $\left\|S\left(x_{1}^{n}\right)\right\| \geq \delta>0$, and fix $x_{i} \in E_{i}$ with $\phi_{i}\left(x_{i}\right)=1$ $(2 \leq i \leq k)$. Then

$$
\left\|T\left(x_{1}^{n}, x_{2}, \ldots, x_{k}\right)\right\|=\left\|S\left(x_{1}^{n}\right)\right\| \geq \delta,
$$

so $T$ is not completely continuous.

Given the spaces $E_{1}, \ldots, E_{k}$, suppose that, for $i \neq j$, every linear operator from $E_{i}$ into $E_{j}^{*}$ is weakly compact. Then, it is known (see [4] for symmetric operators, and [6] for the general case) that, for all $X$, every operator $T \in \mathcal{L}^{k}\left(E_{1}, \ldots, E_{k} ; X\right)$ has a separately $\omega^{*}-\omega^{*}$ continuous extension $\bar{T}$ to the product of the biduals. By Proposition 2.2, all the Aron-Berner extensions of $T$ coincide with $\bar{T}$.

We now consider the polynomial version of the last two results. Unfortunately, we have been unable to obtain them in full generality. 
Proposition 3.8. Suppose $\mathcal{L}\left(E, E^{*}\right)=\mathcal{L}_{\text {wco }}\left(E, E^{*}\right)$, and let $P \in \mathcal{P}\left({ }^{k} E, X\right)$ be a polynomial whose Aron-Berner extension is $X$-valued. Then $P$ is weakly continuous on DP sets.

Proof. Suppose the result is true for all $(k-1)$-homogeneous polynomials, and let $P$ be as in the statement, with associated $T \in \mathcal{L}_{s}^{k}(E, X)$. By the comment above, the extension $\bar{T}$ of $T$ is unique, and therefore symmetric.

For $z \in E^{* *}$, consider the polynomial $z(T) \in \mathcal{P}\left({ }^{k-1} E, X\right)$ given by

$$
z(T)(y)=\bar{T}(y, \stackrel{(k-1)}{\cdots}, y, z) \quad(y \in E) .
$$

The polarization formula implies that $z(T)(y) \in X$. The Aron-Berner extension of $z(T)$ is clearly

$$
\overline{z(T)}(w)=\bar{T}(w, \stackrel{(k-1)}{\cdots}, w, z) \quad\left(w \in E^{* *}\right) .
$$

The polarization formula implies again that $\overline{z(T)}$ is $X$-valued. By the induction hypothesis, $z(T)$ is weakly continuous on DP sets.

The rest of the proof is easily adapted from that of Proposition 3.6.

Corollary 3.9. Suppose $\mathcal{L}\left(E, E^{*}\right)=\mathcal{L}_{\text {wco }}\left(E, E^{*}\right)$. Then, the following assertions are equivalent:

(a) The space E has the DPP;

(b) for all $X$ and $k \in \mathbb{N}$, if the Aron-Berner extension of a polynomial $P \in$ $\mathcal{P}\left({ }^{k} E, X\right)$ is $X$-valued, then $P$ is completely continuous;

(c) for all $X$ and $k \in \mathbb{N}$, every weakly compact $P \in \mathcal{P}\left({ }^{k} E, X\right)$ is completely continuous;

(d) there is $k \in \mathbb{N}$ such that every weakly compact $P \in \mathcal{P}\left({ }^{k} E, c_{0}\right)$ is completely continuous.

We do not know the answer to the following:

Question 3.10. Given a polynomial $P \in \mathcal{P}\left({ }^{k} E, X\right)$, let $T \in \mathcal{L}_{s}^{k}(E, X)$ be its associated symmetric $k$-linear operator. Assume that the Aron-Berner extension of $P$ is $X$-valued. Does $T$ have at least one $X$-valued Aron-Berner extension?

Recall that, if $E^{*}$ has the DPP, then so does $E$ [24, p. 136]. We shall now extend to the multilinear case the following result characterizing the DPP of a dual Banach space:

Proposition 3.11. [10, Proposition 10] A dual Banach space $E^{*}$ has the DPP if and only if, for all $X$ and $T \in \mathcal{L}_{\text {wco }}(E, X)$, the bitranspose $T^{* *}$ is completely continuous.

Proposition 3.12. Let $E_{1}, \ldots, E_{k}$ be Banach spaces such that, for every $i \neq j$, $\mathcal{L}\left(E_{i}, E_{j}^{*}\right)=\mathcal{L}_{\text {wco }}\left(E_{i}, E_{j}^{*}\right)$. The following assertions are equivalent:

(a) $E_{1}^{*}, \ldots, E_{k}^{*}$ have the DPP;

(b) given $X$, let $T \in \mathcal{L}^{k}\left(E_{1}, \ldots, E_{k} ; X\right)$ be an operator; if the Aron-Berner extension $\bar{T}$ of $T$ is $X$-valued, then $\bar{T}$ is completely continuous;

(c) for all $X$, and every weakly compact operator $T \in \mathcal{L}^{k}\left(E_{1}, \ldots, E_{k} ; X\right)$, the Aron-Berner extension $\bar{T}$ of $T$ is completely continuous. 
Proof. (a) $\Rightarrow(\mathrm{b})$ : Suppose $E_{1}^{*}, \ldots, E_{k}^{*}$ have the DPP and let $X$ be a Banach space. We proceed by induction on $k$. If $k=1$, the result follows from Proposition 3.11. Suppose it is true for all $(k-1)$-linear operators and choose an operator $T \in \mathcal{L}^{k}\left(E_{1}, \ldots, E_{k} ; X\right)$. Let $\bar{T}$ be the unique Aron-Berner extension of $T$. For each $z_{k} \in E_{k}^{* *}$, consider the mapping $(\bar{T})_{z_{k}} \in \mathcal{L}^{k-1}\left(E_{1}^{* *}, \ldots, E_{k-1}^{* *} ; X\right)$ defined by

$$
(\bar{T})_{z_{k}}\left(z_{1}, \ldots, z_{k-1}\right)=\bar{T}\left(z_{1}, \ldots, z_{k}\right) \quad\left(z_{i} \in E_{i}^{* *}, \quad 1 \leq i \leq k-1\right) .
$$

Clearly, $(\bar{T})_{z_{k}}$ is the Aron-Berner extension of the $(k-1)$-linear operator $\overline{z_{k}}(T)$ and it is $X$-valued, since $\bar{T}$ is $X$-valued. By the induction hypothesis, $(\bar{T})_{z_{k}}$ is completely continuous. For every $1 \leq i \leq k$ let now $\left(z_{i}^{n}\right)_{n} \subset E_{i}^{* *}$ be a weak Cauchy sequence. We can define the mapping $S \in \mathcal{L}\left(E_{k} ; c(X)\right)$ by

$$
S\left(x_{k}\right)=\left(\bar{T}\left(z_{1}^{n}, \ldots, z_{k-1}^{n}, x_{k}\right)\right)_{n}=\left(S_{n}\left(x_{k}\right)\right)_{n} \quad\left(x_{k} \in E_{k}\right) .
$$

By Lemma 2.1, we know that

$$
S_{n}^{* *}\left(z_{k}\right)=(\bar{T})_{z_{k}}\left(z_{1}^{n}, \ldots, z_{k-1}^{n}\right) .
$$

Since $(\bar{T})_{z_{k}}$ is completely continuous, for every $z_{k} \in E_{k}^{* *}$, the sequence $\left(S_{n}^{* *}\left(z_{k}\right)\right)_{n}$ is convergent. Therefore, $S$ is weakly compact (Lemma 3.3) and, by the DPP of $E_{k}^{*}, S^{* *}$ is completely continuous. Thus, the sequence $\left(S^{* *}\left(z_{k}^{n}\right)\right)_{n}$ converges to some $w=\left(w_{m}\right)_{m} \in c(X)$ and, proceeding as at the end of Proposition 3.6, we obtain that the sequence $\left(\bar{T}\left(z_{1}^{n}, \ldots, z_{k}^{n}\right)\right)_{n}$ is convergent.

(b) $\Rightarrow$ (c) is clear.

$(c) \Rightarrow$ (a): Suppose one of the dual spaces, say $E_{1}^{*}$, does not have the DPP. By Proposition 3.11, there exist $X$ and an operator $U \in \mathcal{L}_{\text {wco }}\left(E_{1}, X\right)$ such that $U^{* *}$ is not completely continuous. For each $i \in\{2, \ldots, k\}$, choose a nonzero form $\phi_{i} \in E_{i}^{*}$, and define the operator $T \in \mathcal{L}^{k}\left(E_{1}, \ldots, E_{k} ; X\right)$ by

$$
T\left(x_{1}, \ldots, x_{k}\right)=\prod_{i=2}^{k} \phi_{i}\left(x_{i}\right) U\left(x_{1}\right) \quad\left(x_{i} \in E_{i}, \quad 1 \leq i \leq k\right) .
$$

Clearly, $T$ is weakly compact, and its extension is not completely continuous.

Corollary 3.13. Let $E$ be a Banach space such that $\mathcal{L}\left(E, E^{*}\right)=\mathcal{L}_{\mathrm{wco}}\left(E, E^{*}\right)$. Then the following assertions are equivalent:

(a) $E^{*}$ has the DPP;

(b) for all $X$ and $k \in \mathbb{N}$, if the Aron-Berner extension $\bar{P}$ of a polynomial $P \in$ $\mathcal{P}\left({ }^{k} E, X\right)$ is $X$-valued, then $\bar{P}$ is completely continuous;

(c) there exists $k \in \mathbb{N}$ such that, for all $X$, the extension $\bar{P}$ of every weakly compact polynomial $P \in \mathcal{P}\left({ }^{k} E, X\right)$ is completely continuous.

\section{UNCONDITIONALLY CONVERGING OPERATORS}

In this Section, we obtain a multilinear version of Pełczyński's property (V) and show that, as an easy consequence of the results in Section 3, every multilinear operator with an $X$-valued Aron-Berner extension is unconditionally converging.

A formal series $\sum x^{n}$ in a Banach space $E$ is weakly unconditionally Cauchy (w.u.C., for short) if there is $C>0$ such that, for any finite subset $\Delta$ of $\mathbb{N}$ and 
any signs \pm , we have $\left\|\sum_{n \in \Delta} \pm x^{n}\right\| \leq C$. For other equivalent definitions, see [13, Theorem V.6]. The series $\sum x^{n}$ is unconditionally convergent if every subseries is norm convergent. Other equivalent definitions may be seen in [14, Theorem 1.9].

A linear operator between Banach spaces is unconditionally converging if it takes w.u.C. series into unconditionally convergent series. A Banach space $E$ is said to have Pełczyński's property $(V)$ if every unconditionally converging linear operator on $E$ is weakly compact. This property was introduced in [29], where it is shown that $C(K)$ spaces have property $(\mathrm{V})$, and that the dual of a space with property $(\mathrm{V})$ is weakly sequentially complete.

Following [16], we say that $T \in \mathcal{L}^{k}\left(E_{1}, \ldots, E_{k} ; X\right)$ is unconditionally converging if, given w.u.C. series $\sum_{n \in \mathbb{N}} x_{i}^{n}$ in $E_{i}(1 \leq i \leq k)$, the sequence

$$
\left(T\left(s_{1}^{m}, \ldots, s_{k}^{m}\right)\right)_{m}
$$

is norm convergent in $X$, where $s_{i}^{m}=\sum_{n=1}^{m} x_{i}^{n}$. This definition may be adapted to polynomials in an obvious way. Since a linear operator fails to be unconditionally converging if and only if it preserves a copy of $c_{0}$ [13, Exercise V.8], it is clear that the definition of unconditionally converging $k$-linear operators agrees for $k=1$ with that of unconditionally converging linear operators.

By the polarization formula, a polynomial is unconditionally converging if and only if so is its associated symmetric multilinear operator.

A different notion of unconditionally converging multilinear operators and polynomials, giving rise to a strictly wider class, was used in [21] and [20]. It is not possible, however, for this wider class, to establish a multilinear equivalent version of property $(\mathrm{V})$, and this is why we adopt here the definition of [16], which is in some respects quite natural.

Now we can prove a result parallel to Lemma 3.2:

Lemma 4.1. Let $T \in \mathcal{L}^{k}\left(E_{1}, \ldots, E_{k} ; X\right)$. Then $T$ is unconditionally converging if and only if, for all w.u.C. series $\sum_{n \in \mathbb{N}} x_{j}^{n}$ in $E_{j}(1 \leq j \leq k)$, and for all $i \in$ $\{1, \ldots, k\}$, the sequences

$$
\left(T\left(s_{1}^{n}, \ldots, s_{i-1}^{n}, s_{i}^{m}, s_{i+1}^{n}, \ldots, s_{k}^{n}\right)\right)_{m}
$$

converge uniformly on $n$, in the sense that

$$
\lim _{p, q \rightarrow \infty} \sup _{n \in \mathbb{N}}\left\|T\left(s_{1}^{n}, \ldots, s_{i-1}^{n}, s_{i}^{p}-s_{i}^{q}, s_{i+1}^{n}, \ldots, s_{k}^{n}\right)\right\|=0 .
$$

Proof. For simplicity, let us denote

$$
T_{n}\left(s_{i}^{p}-s_{i}^{q}\right)=T\left(s_{1}^{n}, \ldots, s_{i-1}^{n}, s_{i}^{p}-s_{i}^{q}, s_{i+1}^{n}, \ldots, s_{k}^{n}\right) .
$$

Suppose $T$ is unconditionally converging and the result fails. Then we can find w.u.C. series $\sum x_{j}^{n}$ in $E_{j}(1 \leq j \leq k), i \in\{1, \ldots, k\}$, indices $0=n(0)<n(1)<$ $n(2)<\cdots$, and $0=p(0)<q(1)<p(1)<q(2)<p(2)<\cdots$, and $\epsilon>0$, such that

$$
\left\|T_{n(r)}\left(s_{i}^{p(r)}-s_{i}^{q(r)}\right)\right\|>\epsilon \quad(r \in \mathbb{N}) .
$$


Now, for $j \neq i$, we define

$$
y_{j}^{l}=\left\{\begin{array}{cl}
\sum_{m=n((l-1) / 2)+1}^{n((l+1) / 2)} x_{j}^{m} & \text { if } l \text { is odd } \\
0, & \text { if } l \text { is even }
\end{array}\right.
$$

and

$$
y_{i}^{l}= \begin{cases}\sum_{m=p((l-1) / 2)+1}^{q((l+1) / 2)} x_{i}^{m}, & \text { if } l \text { is odd } \\ \sum_{m=q(l / 2)+1}^{p(l / 2)} x_{i}^{m}, & \text { if } l \text { is even. }\end{cases}
$$

Clearly, $\sum_{l \in \mathbb{N}} y_{j}^{l}$ is w.u.C. for all $j \in\{1, \ldots, k\}$. Letting

$$
\sigma_{j}^{r}=\sum_{l=1}^{r} y_{j}^{l},
$$

it is easy to check that, whenever $r$ is odd, we have

$$
\left\|T\left(\sigma_{1}^{r+1}, \ldots \sigma_{k}^{r+1}\right)-T\left(\sigma_{1}^{r}, \ldots, \sigma_{k}^{r}\right)\right\|=\left\|T_{n((r+1) / 2)}\left(s_{i}^{p((r+1) / 2)}-s_{i}^{q((r+1) / 2)}\right)\right\|>\epsilon,
$$

so the sequence $\left(T\left(\sigma_{1}^{r}, \ldots, \sigma_{k}^{r}\right)\right)_{r}$ is not Cauchy, a contradiction.

For the converse, suppose $T$ is not unconditionally converging. Then there exist $\epsilon>0$, w.u.C. series as in the statement, and two increasing sequences of indices $(p(r))_{r}$ and $(q(r))_{r}$, with $p(0)=q(0)=0$, such that

$$
\left\|T\left(s_{1}^{p(r)}, \ldots, s_{k}^{p(r)}\right)-T\left(s_{1}^{q(r)}, \ldots, s_{k}^{q(r)}\right)\right\|>\epsilon .
$$

On the other hand, we can write

$$
\begin{aligned}
& \left\|T\left(s_{1}^{p(r)}, \ldots, s_{k}^{p(r)}\right)-T\left(s_{1}^{q(r)}, \ldots, s_{k}^{q(r)}\right)\right\| \leq \\
& \quad\left\|T\left(s_{1}^{p(r)}-s_{1}^{q(r)}, s_{2}^{p(r)}, \ldots, s_{k}^{p(r)}\right)\right\|+\left\|T\left(s_{1}^{q(r)}, s_{2}^{p(r)}-s_{2}^{q(r)}, s_{3}^{p(r)}, \ldots, s_{k}^{p(r)}\right)\right\| \\
& \quad+\cdots+\left\|T\left(s_{1}^{q(r)}, \ldots, s_{k-1}^{q(r)}, s_{k}^{p(r)}-s_{k}^{q(r)}\right)\right\| .
\end{aligned}
$$

We claim that each term of the right hand side can be made as small as we wish, for $r$ large enough. This is in contradiction with (5). As an example, we prove the claim for the second term. Define

$$
\begin{aligned}
& y_{1}^{n}=\sum_{m=q(n-1)+1}^{q(n)} x_{1}^{m}, \\
& y_{2}^{n}=x_{2}^{n},
\end{aligned}
$$




$$
y_{j}^{n}=\sum_{m=p(n-1)+1}^{p(n)} x_{j}^{m}, \quad \text { if } j \geq 3 .
$$

Clearly, the series $\sum_{n} y_{j}^{n}$ is w.u.C. for each $j$. Therefore, by our hypothesis,

$$
\begin{aligned}
& \lim _{r \rightarrow \infty} \| T\left(s_{1}^{q(r)}, s_{2}^{p(r)}-s_{2}^{q(r)}, s_{3}^{p(r)}, \ldots, s_{k}^{p(r)}\right) \|= \\
& \lim _{r \rightarrow \infty}\left\|T\left(\sum_{n=1}^{r} y_{1}^{n}, \sum_{n=1}^{p(r)} y_{2}^{n}-\sum_{n=1}^{q(r)} y_{2}^{n}, \sum_{n=1}^{r} y_{3}^{n}, \ldots, \sum_{n=1}^{r} y_{k}^{n}\right)\right\|=0,
\end{aligned}
$$

which finishes the proof.

Now, using Lemma 4.1, property (V), and partial sums of w.u.C. series, instead of Lemma 3.2, the RDPP, and weak Cauchy sequences, the following analogs of Theorem 3.4 and Corollary 3.5 can be obtained with similar proofs.

Theorem 4.2. The following assertions are equivalent:

(a) The spaces $E_{1}, \ldots, E_{k}$ have property $(V)$;

(b) for all $X$ and each unconditionally converging $T \in \mathcal{L}^{k}\left(E_{1}, \ldots, E_{k} ; X\right)$, every Aron-Berner extension of $T$ is $X$-valued;

(c) for all $X$, every unconditionally converging $T \in \mathcal{L}^{k}\left(E_{1}, \ldots, E_{k} ; X\right)$ has an $X$-valued Aron-Berner extension.

Note that, since the dual of a space with property $(\mathrm{V})$ is weakly sequentially complete, we have $\mathcal{L}\left(E_{i}, E_{j}^{*}\right)=\mathcal{L}_{\text {wco }}\left(E_{i}, E_{j}^{*}\right)$ for all $i, j$, under any of the equivalent assertions of the above Theorem, so each multilinear operator has a unique AronBerner extension.

Corollary 4.3. The following assertions are equivalent:

(a) The space E has property (V);

(b) for all $X$ and $k \in \mathbb{N}$, the Aron-Berner extension of every unconditionally converging $P \in \mathcal{P}\left({ }^{k} E, X\right)$ is $X$-valued;

(c) there is $k \in \mathbb{N}$ such that, for all $X$, the Aron-Berner extension of every unconditionally converging $P \in \mathcal{P}\left({ }^{k} E, X\right)$ is $X$-valued.

We now show that a multilinear operator which is weakly continuous on DP sets is also unconditionally converging. Easily, the result is true for polynomials as well.

Proposition 4.4. If $T \in \mathcal{L}^{k}\left(E_{1}, \ldots, E_{k} ; X\right)$ is weakly continuous on DP sets, then $T$ is unconditionally converging.

Proof. Take w.u.C. series $\sum_{n} x_{i}^{n}$ in $E_{i}(1 \leq i \leq k)$. We can define linear operators $S_{i}: c_{0} \rightarrow E_{i}$ by $S_{i}\left(e_{n}\right)=x_{i}^{n}$, where $\left(e_{n}\right)$ is the unit vector basis of $c_{0}$. Since the unit ball of $c_{0}$ is a DP set, the sequence $\left(\sum_{j=1}^{n} e_{j}\right)_{n}$ is a weak Cauchy DP sequence, and so is the sequence

$$
\left(\sum_{j=1}^{n} x_{i}^{j}\right)_{n}=\left(S_{i}\left(\sum_{j=1}^{n} e_{j}\right)\right)_{n}
$$


for each $1 \leq i \leq k$. Hence, the sequence

$$
\left(T\left(\sum_{j=1}^{n} x_{1}^{j}, \ldots, \sum_{j=1}^{n} x_{k}^{j}\right)\right)_{n}
$$

converges in $X$.

Proposition 4.5. If $T \in \mathcal{L}^{k}\left(E_{1}, \ldots, E_{k} ; X\right)$ has an $X$-valued Aron-Berner extension, then $T$ is unconditionally converging. If the Aron-Berner extension of $P \in \mathcal{P}\left({ }^{k} E, X\right)$ is $X$-valued, then $P$ is unconditionally converging.

Proof. For the multilinear case, use Propositions 3.6 and 4.4. Let $P \in \mathcal{P}\left({ }^{k} E, X\right)$ so that $\bar{P}$ is $X$-valued. Take a w.u.C. series $\sum x_{n}$ in $E$. Define $S: c_{0} \rightarrow E$ by $S\left(e_{n}\right)=x_{n}$. Then $P \circ S \in \mathcal{P}\left({ }^{k} c_{0}, X\right)$ and its extension $\overline{P \circ S}=\bar{P} \circ S^{* *}$ is $X$-valued. By Corollary 3.9, $P \circ S$ is completely continuous. Therefore, the sequence

$$
\left(P \circ S\left(\sum_{j=1}^{n} e_{j}\right)\right)_{n}=\left(P\left(\sum_{j=1}^{n} x_{j}\right)\right)_{n}
$$

is norm convergent in $X$.

Arguing as in the case of completely continuous operators, it is easy to prove the following results parallel to Proposition 3.12 and Corollary 3.13.

Proposition 4.6. Let $E_{1}, \ldots, E_{k}$ be Banach spaces such that, for every $i \neq j$, we have $\mathcal{L}\left(E_{i}, E_{j}^{*}\right)=\mathcal{L}_{\mathrm{wco}}\left(E_{i}, E_{j}^{*}\right)$. Then, for all $X$, whenever the Aron-Berner extension $\bar{T}$ of $T \in \mathcal{L}^{k}\left(E_{1}, \ldots, E_{k} ; X\right)$ is $X$-valued, $\bar{T}$ is unconditionally converging.

Corollary 4.7. Let $E$ be a Banach space such that $\mathcal{L}\left(E, E^{*}\right)=\mathcal{L}_{\text {wco }}\left(E, E^{*}\right)$. Then, for all $X$ and $k \in \mathbb{N}$, whenever the Aron-Berner extension $\bar{P}$ of a polynomial $P \in$ $\mathcal{P}\left({ }^{k} E, X\right)$ is $X$-valued, $\bar{P}$ is unconditionally converging.

We do not know if the condition on weak compactness of the linear operators may be dropped in Propositions 3.12 and 4.6, and their Corollaries.

We finish the section with the following result, which is also true for polynomials:

Corollary 4.8. Suppose the spaces $E_{1}, \ldots, E_{k}$ have property $(V)$ and the DPP. For an operator $T \in \mathcal{L}^{k}\left(E_{1}, \ldots, E_{k} ; X\right)$, the following assertions are equivalent:

(a) $T$ is completely continuous;

(b) $T$ is unconditionally converging;

(c) $\bar{T}$ is $X$-valued.

Assume moreover that $E_{1}^{*}, \ldots, E_{k}^{*}$ have the DPP. Then the above assertions are also equivalent to:

(d) $\bar{T}$ is completely continuous;

(e) $\bar{T}$ is unconditionally converging;

Proof. Note first that the Aron-Berner extension $\bar{T}$ of $T$ is unique, since the spaces have property (V). Clearly, (a) $\Rightarrow(\mathrm{b})$, and $(\mathrm{d}) \Rightarrow(\mathrm{e}) \Rightarrow(\mathrm{b})$. By Theorem $4.2,(\mathrm{~b}) \Rightarrow$ (c). By Theorem 3.7, (c) $\Rightarrow$ (a). If the duals have the DPP, from Proposition 3.12, we obtain $(\mathrm{c}) \Rightarrow(\mathrm{d})$. 
Many spaces satisfy all the requirements of Corollary 4.8. For instance, every $C(K)$ space, every $C^{*}$-algebra whose dual has the DPP [28], the space $H^{\infty}[7,8]$, the disc algebra $[12,32]$, the space of analytic uniformly convergent Fourier series on the unit circle [33].

\section{EXTENSION OF HOLOMORPHIC MAPPINGS}

Suppose now that $E$ and $X$ are complex Banach spaces. We denote by $\mathcal{H}_{b}(E, X)$ the space of holomorphic mappings of bounded type from $E$ into $X$. Many authors have studied this space. We refer to [26] for some basic properties. Let $f \in \mathcal{H}_{b}(E, X)$ be given, with Taylor series expansion at the origin $f=\sum_{k=0}^{\infty} P_{k}$, where $P_{k} \in$ $\mathcal{P}\left({ }^{k} E, X\right)$. Then $f$ has an $X$-valued Aron-Berner extension $\bar{f} \in \mathcal{H}_{b}\left(E^{* *}, X\right)$ if and only if $\bar{P}_{k}$ is $X$-valued for all $k \in \mathbb{N}$ [18, Theorem 3.3].

We can define in an obvious way the weakly compact (resp. completely continuous, unconditionally converging, etc.) holomorphic mappings of bounded type.

The mapping $f$ is weakly compact (resp. weakly continuous on DP sets, completely continuous) if and only if so is $P_{k}$ for each $k \in \mathbb{N}$ [31] (resp. [19, 2]).

It is easy to see that $f$ is unconditionally converging if and only if so is $P_{k}$ for each $k \in \mathbb{N}$. Indeed, for the "only if" part, take a w.u.C. series $\sum x_{i}$ in $E$. Let $s_{n}=\sum_{i=1}^{n} x_{i}$. Then $\left(f\left(s_{n}\right)\right)_{n}$ is convergent. Given $\epsilon>0$, since $f$ is uniformly continuous on bounded sets, we can find $\delta>0$ such that

$$
\left\|f\left(\lambda s_{n}\right)-f\left(\mu s_{n}\right)\right\|<\epsilon / 3 \quad(n \in \mathbb{N})
$$

for all complex $\lambda, \mu$ with $|\lambda|=|\mu|=1$ and $|\lambda-\mu|<\delta$. Using compactness of the unit circle in the complex plane, we get

$$
\left\|f\left(\lambda s_{n}\right)-f\left(\lambda s_{m}\right)\right\|<\epsilon \quad(|\lambda|=1)
$$

for $n, m$ large enough. Fix $k \in \mathbb{N}$. As in [2, p. 59], the Cauchy integral formula yields

$$
\left\|P_{k}\left(s_{n}\right)-P_{k}\left(s_{m}\right)\right\|<\epsilon
$$

for large $n, m$. The converse is standard.

Therefore, we can immediately extend the results obtained in the previous sections of this paper from polynomials to holomorphic mappings. For instance, the extension of Corollary 4.3 will be as follows:

The space $E$ has property $(V)$ if and only if for every $X$ and every unconditionally converging $f \in \mathcal{H}_{b}(E, X)$, the Aron-Berner extension of $f$ is $X$-valued.

\section{AN OPEN PROBLEM}

It would be very interesting to describe the multilinear operators with " $X$-valued" extensions. For simplicity, we discuss the subject in the polynomial setting.

If $P_{1} \in \mathcal{P}\left({ }^{k} E, X\right)$ is weakly compact, then there are a reflexive Banach space $Y_{1}$, a polynomial $Q_{1} \in \mathcal{P}\left({ }^{k} E, Y_{1}\right)$ and an operator $S_{1} \in \mathcal{L}\left(Y_{1}, X\right)$ so that $P_{1}=S_{1} \circ Q_{1}$ [31] and, clearly, $\overline{P_{1}}$ is $X$-valued.

We say that a polynomial $P_{2} \in \mathcal{P}\left({ }^{k} E, X\right)$ is regular if there are a reflexive Banach space $Y_{2}$, an operator $S_{2} \in \mathcal{L}\left(E, Y_{2}\right)$ and a polynomial $Q_{2} \in \mathcal{P}\left({ }^{k} Y_{2}, X\right)$ so that 
$P_{2}=Q_{2} \circ S_{2}$. Then $P_{2}$ need not be weakly compact, but still $\overline{P_{2}}$ is $X$-valued. Regular polynomials have been studied in [23]. There are many regular polynomials which are not weakly compact. Indeed, it is shown in the proof of [21, Proposition 14] that, whenever $E$ has a quotient isomorphic to $\ell_{2}$ (in particular, if $E$ contains a copy of $\ell_{1}$ ) and $X$ is non-reflexive, there are regular polynomials of every degree from $E$ into $X$ which are not weakly compact.

Clearly, if a polynomial $P$ may be written in the form $P_{1}+P_{2}$, with $P_{1}$ weakly compact and $P_{2}$ regular, then $\bar{P}$ is $X$-valued. We do not know if the converse is true, in other words, if every polynomial $P \in \mathcal{P}\left({ }^{k} E, X\right)$ whose Aron-Berner extension is $X$-valued may be decomposed into a sum of a weakly compact polynomial and a regular polynomial. In the affirmative, the fact of having an $X$-valued Aron-Berner extension would be closely connected to factorizing in some way through a reflexive Banach space and hence to weak compactness.

The authors are grateful to Ignacio Zalduendo for helpful discussions. The second named author also thanks Fernando Bombal for help and advice.

\section{REFERENCES}

[1] K. T. Andrews, Dunford-Pettis sets in the space of Bochner integrable functions, Math. Ann. 241 (1979), 35-41.

[2] R. M. Aron, Weakly uniformly continuous and weakly sequentially continuous entire functions, in: J. A. Barroso (ed.), Advances in Holomorphy, Math. Studies 34, North-Holland, Amsterdam 1979, 47-66.

[3] R. M. Aron and P. D. Berner, A Hahn-Banach extension theorem for analytic mappings, Bull. Soc. Math. France 106 (1978), 3-24.

[4] R. M. Aron, B. J. Cole, and T. W. Gamelin, Spectra of algebras of analytic functions on a Banach space, J. Reine Angew. Math. 415 (1991), 51-93.

[5] R. M. Aron, C. Hervés, and M. Valdivia, Weakly continuous mappings on Banach spaces, J. Funct. Anal. 52 (1983), 189-204.

[6] F. Bombal and I. Villanueva, Multilinear operators on spaces of continuous functions, Funct. Approx. Comment. Math. 26 (1998), 117-126.

[7] J. Bourgain, $H^{\infty}$ is a Grothendieck space, Studia Math. 75 (1983), 193-216.

[8] J. Bourgain, New Banach space properties of the disc algebra and $H^{\infty}$, Acta Math. 152 (1984), $1-48$.

[9] D. Carando, Extendible polynomials on Banach spaces, J. Math. Anal. Appl. 233 (1999), 359-372.

[10] J. M. F. Castillo and M. González, New results on the Dunford-Pettis property, Bull. London Math. Soc. 27 (1995), 599-605.

[11] A. M. Davie and T. W. Gamelin, A theorem on polynomial-star approximation, Proc. Amer. Math. Soc. 106 (1989), 351-356.

[12] F. Delbaen, Weakly compact operators on the disc algebra, J. Algebra 45 (1977), 284-294.

[13] J. Diestel, Sequences and Series in Banach Spaces, Graduate Texts in Math. 92, Springer, Berlin 1984.

[14] J. Diestel, H. Jarchow, and A. Tonge, Absolutely Summing Operators, Cambridge Stud. Adv. Math. 43, Cambridge Univ. Press, Cambridge 1995.

[15] S. Dineen, Complex Analysis on Infinite Dimensional Spaces, Springer Monogr. Math., Springer, Berlin 1999.

[16] M. Fernández Unzueta, Unconditionally convergent polynomials in Banach spaces and related properties, Extracta Math. 12 (1997), 305-307. 
[17] J. Ferrera, J. Gómez, and J. G. Llavona, On completion of spaces of weakly continuous functions, Bull. London Math. Soc. 15 (1983), 260-264.

[18] P. Galindo, D. Garcí a, M. Maestre, and J. Mujica, Extension of multilinear mappings on Banach spaces, Studia Math. 108 (1994), 55-76.

[19] M. González and J. M. Gutiérrez, Weakly continuous mappings on Banach spaces with the Dunford-Pettis property, J. Math. Anal. Appl. 173 (1993), 470-482.

[20] M. González and J. M. Gutiérrez, When every polynomial is unconditionally converging, Arch. Math. 63 (1994), 145-151.

[21] M. González and J. M. Gutiérrez, Unconditionally converging polynomials on Banach spaces, Math. Proc. Cambridge Philos. Soc. 117 (1995), 321-331.

[22] M. González and J. M. Gutiérrez, Polynomial Grothendieck properties, Glasgow Math. J. 37 (1995), 211-219.

[23] M. González and J. M. Gutiérrez, Injective factorization of holomorphic mappings, Proc. Amer. Math. Soc. 127 (1999), 1715-1721. See also Erratum in vol. 129 (2001), 1255-1256.

[24] A. Grothendieck, Sur les applications linéaires faiblement compactes d'espaces du type $C(K)$, Canad. J. Math. 5 (1953), 129-173.

[25] A. Grothendieck, Topological Vector Spaces, Notes Math. Appl., Gordon and Breach, New York 1975.

[26] J. M. Isidro, Topological duality on the functional space $\left(\mathcal{H}_{b}(U, F), \tau_{b}\right)$, Proc. Roy. Irish Acad. 79A (1979), 115-130.

[27] J. Mujica, Complex Analysis in Banach Spaces, Math. Studies 120, North-Holland, Amsterdam 1986.

[28] H. Pfitzner, Weak compactness in the dual of a $C^{*}$-algebra is determined commutatively, Math. Ann. 298 (1994), 349-371.

[29] A. Pełczyński, Banach spaces on which every unconditionally converging operator is weakly compact, Bull. Acad. Polon. Sci. Sér. Sci. Math. Astr. Phys. 10 (1962), 641-648.

[30] R. A. Ryan, Dunford-Pettis properties, Bull. Acad. Polon. Sci. Sér. Sci. Math. 27 (1979), 373-379.

[31] R. A. Ryan, Weakly compact holomorphic mappings on Banach spaces, Pacific J. Math. 131 (1988), 179-190.

[32] E. Saab and P. Saab, On stability problems of some properties in Banach spaces, in: K. Sarosz (ed.), Function Spaces, Lecture Notes Pure Appl. Math. 136, Dekker, New York 1992, 367-394.

[33] S. F. Saccone, The Pełczyński property for tight subspaces, J. Funct. Anal. 148 (1997), 86116.

[34] I. Villanueva, Completely continuous multilinear operators on $C(K)$ spaces, Proc. Amer. Math. Soc. 128 (2000), 793-801.

[35] I. Zalduendo, A canonical extension for analytic functions on Banach spaces, Trans. Amer. Math. Soc. 320 (1990), 747-763.

[36] I. Zalduendo, Extending polynomials - A survey, Publ. Dep. Análisis Matemático, Universidad Complutense de Madrid, Madrid 1998.

Departamento de Matemáticas, ets de Ingenieros Industriales, Universidad Politécnica de Madrid, C. José Gutiérrez Abascal 2, 28006 Madrid (Spain)

E-mail address: gutierrezj@member.ams.org

Departamento de Análisis Matemático, Facultad de Matemáticas, Universidad Complutense de Madrid, 28040 Madrid (Spain)

E-mail address: ignaciov@mat.ucm.es 